\title{
Automatic Electromechanical Single Plate Clutch with Overload Protection Roller Clutch for Machine Safety
}

\author{
Lokesh V. Pithe* and Avinash M. Badadhe ${ }^{\#}$ \\ *\#JSPM's College of Engineering, Pune 411033, Maharashtra, India
}

(Received 21 January 2021; Accepted 25 March 2021)

https://doi.org/10.36224/ijes.140104

\begin{abstract}
This paper summarizes the development of the automatic Electro-Mechanical single plate clutch with the overload protection roller clutch. The major challenges in a Single Plate Clutch automation are the clutch control, which is presently manual. It increases the manual effort and complication during its operation. Due to the complex structure and high cost, the clutch control system used in an advanced vehicle cannot use in economical Vehicles. This situation forces us to develop a Special electromechanical clutch that controls automatically. The rarely occurring overload on machines, create some malfunctioning in the System, which may damage its members. By using an overload protection clutch, such overload can neutralize. We develop an Automatic Engage-disengage Overload Protection Roller Clutch. The facility provided to change its torque carrying capacity make this roller clutch special. Thus the combination of the automated single-plate clutch and the overload protection clutch is a desirable one and also essential. After the market survey, it observed that there is no such combined system is available. If we install these two devices separately, it will require more space and cost, which is infeasible.
\end{abstract}

Keywords: Roller Clutch, Overload Protection, Single Plate Clutch, Varying Torque Capacity.

\section{Introduction}

\subsection{Automatic Single Plate Clutch}

Frictional clutch: Conventional transmission system uses a single plate clutch with a manual transmission gearbox. The auto-disengagement single-clutch employs only one set of compression springs instead of the usual two-set, such a construction it is possible to reduce the weights of the centrifugal member. When the clutch disengaged during the engine is running at idling speed, the hinged bob-weights rest against the lower or inward sides of holes in the flywheel. When the engine accelerated above 700 to $800 \mathrm{rpm}$, the weights fly outwards cause to their lever end compress the springs to engage the clutch plates. When the full engagement pressure between the clutch members has attained, any further increase in engine speed brings limiting the pressure plate in action. This construction keeps the clutch normally disengaged condition, unlike in the conventional that is in engaged condition, which needs manual lever to disengage.

The conventional clutches use a full ace lining, meaning that the entire face of the clutch plate lined with friction material. Conventionally the friction lining material used is asbestos base. A friction clutch has its Principal application in the transmission of the power of shafts and machines, which must be started and stopped frequently. The force of friction used to start the driven shaft from rest and gradually brings it up to the proper speed without excessive slipping of the friction surfaces. In automobiles, the friction clutch used to connect the engine to the drive shaft. 


\subsection{Torque Limiters}

The torque limiter is a system that protects the machines or their sub-assembly from the overload adverse impact. It disengages the system or transmitting the motion with slipping like a clutch. Because of torque limiter, jamming or damaging of machines can be avoided. Torque limiter controls the amount of torque to the predetermined level. It may shut off the system or dissipate energy like frictional clutches without malfunctioning or blockage of the machine.

By using an electronic motor that has a current limitation, torque overload is possible to control. Due to this, many mechanical torque limiters are outdated. Moreover, when the machine is dynamic, also the calculation of inertia of the moving part is complicated, this Mechanical torque limiter may work. An example is by using breaking system rotating mass can abruptly decelerate faster than the drive generally accelerates, which will create torque overload with the help of reflected inertia. The reflected inertia and electronic system are independent, will easily overcome the peak torque value of the motor. Because of advanced torque limiter having good sensitivity and accuracy, it removes the consideration of the older and bulkier design of torque limiter. Newly design torque limiter work with very less impact on mass, size, balance, and power consumption. There are two types of torque limiters. In slip type, torque limiters drive shaft rotates at a faster speed than the driven shaft. This limiter slips at predetermine design torque setting value and again reengage as soon as torque reduces below the design limit.

\section{Literature Review}

Guy James Burlington [1], He did a very close and detailed invention regarding the clutch and its mechanism. Many times in vacuum cleaner with the help of cleaner head or floor tool, dirty air sucked. For this, the motor is used, which is driven by the Fan. The device such as the brush bar is located at the entry of dirty air inlet so that it activates fibers of carpet on which vacuum cleaner moves. Some obstacles may come due to objects which on floor surface jam the Inlet of a vacuum cleaner and hence vacuum motor goes under overload situation and may get burned or damaged. When relative speed exceeds above predetermine value with the help of actuators, torque gets controlled and avoids the burning of the motor.

Nicolae Eftimie [3], After the numerical simulation experimentation by considering kinematic and dynamic modeling, he concludes that the parameter which effects on ball clutch is the ratio between the inertia moments at the driven and driving parts, spring type, its rigidity and pretension spring force. Appropriate balancing of inertia moment of driven parts causes to sustain moderate shock value. It causes to avoid the problems when the clutch is assembled in the body pass through frequently under striking. In the end, by keeping characteristics of safety clutch in mind, kinematic and dynamic optimization, final computer simulation presented.

Duane W. Woltjen [4], He did an invention related to torque-limiting overload coupling due to this coupling as soon as the force on the shaft goes on beyond the design Torque, coupling prevents the rotation of the shaft. Rotating and rotatable parts assembled intelligently such that it can be easily adjusted to assumed torque correctly before disengagement of device occur and hence used as a safety device in industries and robotics.

Donald L. Miller [5], He did an invention in electric overload clutch, he presented that due to usage of the electromagnetic clutch which has over-torque triggered release capacity there is no need to reduce clutch speed to reengage the coupling. Due to overload in spring, the electromagnetic force gets reversed. It caused to balance the pressure of both sides by keeping both stationary and movable torque transmitting part away from each other. The electric switches have the advantage of changing current flow in the electromagnetic clutch. 
J. Mech. Des, Described that under misalignment, the Teeth of a coupling hub and sleeve slide axially over each other. This motion and torque transmission generate friction forces. Though laboratory measurements indicated that, friction coefficients are very low, many of thrust bearing failure blamed on coupling "lock-up." This paper discusses the results obtained from an exhaustive test program in which the friction forces were measure as a function of many variables, including torque, axial velocity, misalignment, and lubrication. Friction coefficients as more as were computed when the coupling operated under adverse conditions. Lubrication proved to have a considerable influence on the magnitude of the friction coefficients.

M. M. Calistrat, Explained what causes failure in gear couplings. Speed having a significant influence on coupling Wear. Higher Speed of coupling reduces the wear-rate while the low Speeds, with moderate lubricants, causes coupling failure. We can only conclude that increasing rotation speed helps in improving the lubrication conditions. Worm tracks caused by vary localized welding and the sliding velocity alone cannot blame for this type of failure.

\section{Literature Gap}

After careful review of the literature on the overload protection clutches, it is clear that the combination of the automated clutch and the overload protection clutch is a desirable one and also essential. It has observed that no such integral devices are available in the market and also that if these two elements installed separated will Increase the space needed, to cause an increased cost. Also, the ball clutch Unit is engaged and disengage only at one single set torque but need to operate the ball clutch at different torque value. Present Automatic clutches are more costly, Complex, and require high maintenance.

\section{Problem Statement}

The different automatic clutch control mechanisms discussed below:

\subsection{Magnetic clutches}

Magnetic clutches are somewhat similar to the conventional plate clutches but with the difference that the clutching and declutching operations governed by an electromagnetic force. These clutches are available in two modes, fluid magnetic clutches, and powder magnetic clutch.

\subsection{Fluid magnetic clutches}

The fluid magnetic clutches develop electromagnetic force required for clutch operation using magnetic fluid. Extremely compactness is the main advantage of these clutches. It also has good power to weight ratio. Because higher cost these clutches application is restricted to few modern automobiles.

\subsection{Powder magnetic clutches}

The powder magnetic clutches, though low at cost, have a very high weight to power ratio makes them bulky. Because of the consumption of a considerable amount of space, they find applications only in stationary applications like machine tools and not mobile applications like automobiles.

\subsection{Centrifugal clutches}


Because of the limitation of the torque transmitting capacity of the centrifugal clutch, these clutches not possible to use in low, medium, or heavy commercial vehicles. These clutches useful in mopeds and scooters where limited space is available.

When output shaft undergoes overload, it causes the burning of the motor or stuck the engine. Sometimes overload also damages the drive component or other parts related to sub-assembly. Hence it is necessary to disconnect output and input shaft when output shaft undergoes overload. The ball clutch isolates the input driver member from the output shaft and avoid further possible damage.

\section{Proposed methodology}

\subsection{Theoretical Work}

i) Find out the literature gap and define problem statements after studying the various configuration of the test rig. For this taking reference from different handbooks, patent documents, and technical research papers.

ii) Find out the solution to the problem.

\subsection{Design and Development}

Design of the entire system, Dimensions, theoretical derivations of single plate clutch, clutch control components and overload protection clutch using cad software and then validation of result using Ansys workbench.

\subsection{Fabrication}

Fabricate the component using an appropriate manufacturing method then assemble the parts for the test setup.

\section{Construction}

The 50-Watt Motor is attached to the Drive Pulley, through the rubber belt. Drive pulley is attaché to the input shaft. The Flywheel mounted on the input shaft. The Flywheel also supported by the main shaft. The clutch plate mounted on the main shaft sandwich in between flywheel and pressure plate. One end of the screw is fixed to the pressure plate while its thread interlock to the inner threaded surface of the spur gear. Teeth of spur gear engaged with another small spur gear, which attached to the 12 volt DC motor. The main shaft passes through the screw to attach the slot flange of an overload protection clutch. Three balls, springs, plungers arranged in series and placed in the holes of the cylindrical body. Cylindrical body enclose by casing. Torque capability adjustment nut is attached to the output shaft through threading. The Cylindrical body, Casing, and Nut mounted on the output shaft. Small end testing pulley attaché to the output shaft. All shaft supported by ball bearings. The whole power train mounted on the frame.

\section{Working}

The regulator is attached to the motor. According to our needs, the motor speed changed by the regulator. Motor power is transfer to the pulley via the belt. Pulley rotates the flywheel through the input shaft. During engage condition, power then transferred to the clutch plate. A Clutch plate, having a lining of friction material Ferodo, which transfers power to the pressure plate and then to the output shaft of the 
clutch. DC motor rotates the spur gear in both rotational directions. The screw moves forward or backward according to the direction of rotation of spur gear. To stop the rotation of the screw stopper is provided.

Power continuously transfers through the main shaft to the overload protection clutch. The pressure exerted by the springs on the roller (ball) to lock in the slots of the flange cause to rotate the cylindrical body, which further transfer power to the output shaft. This an engaged condition of a safety clutch. During overload occur on the output shaft, the slot flange exerts pressure on the rollers on the opposite side of the spring pressure. If this pressure exceeds spring pressure, roller slip from the slot and moves to consecutive next slot and again slip. This action repeats until the overload removed. This position is the disengage position of a safety clutch.

Spring tension adjusting nut provided, to vary the torque carrying capacity of safety clutch. Each rotation of the nut moves Casing forward or backward according to the direction of rotation of the nut. Forward movement of Casing pushes the plunger forward. The further plunger pushes spring to stuck rollers more tightly. Due to this torque carrying capacity of clutch is increased whereas, the reverse rotation of nut reduces the torque carrying capacity of a safety clutch.

\section{Three $D$ modelling of power train}

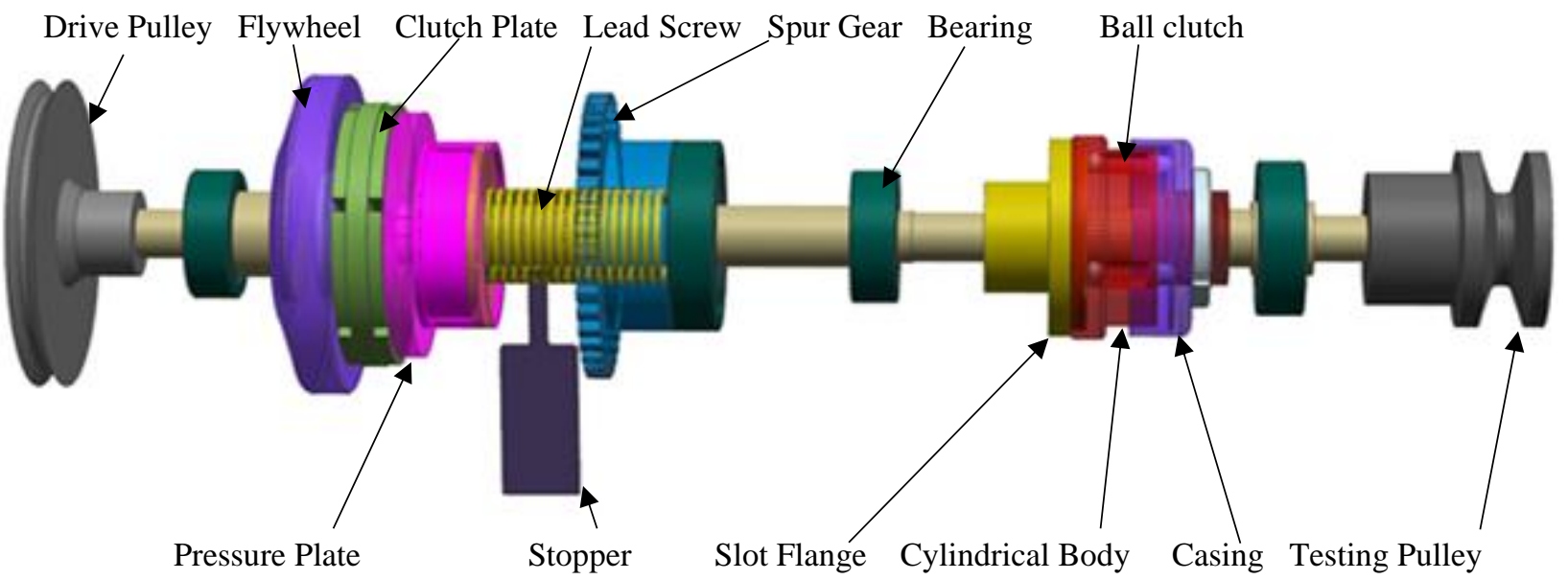

Figure 1: 3 D Modelling of Power Train

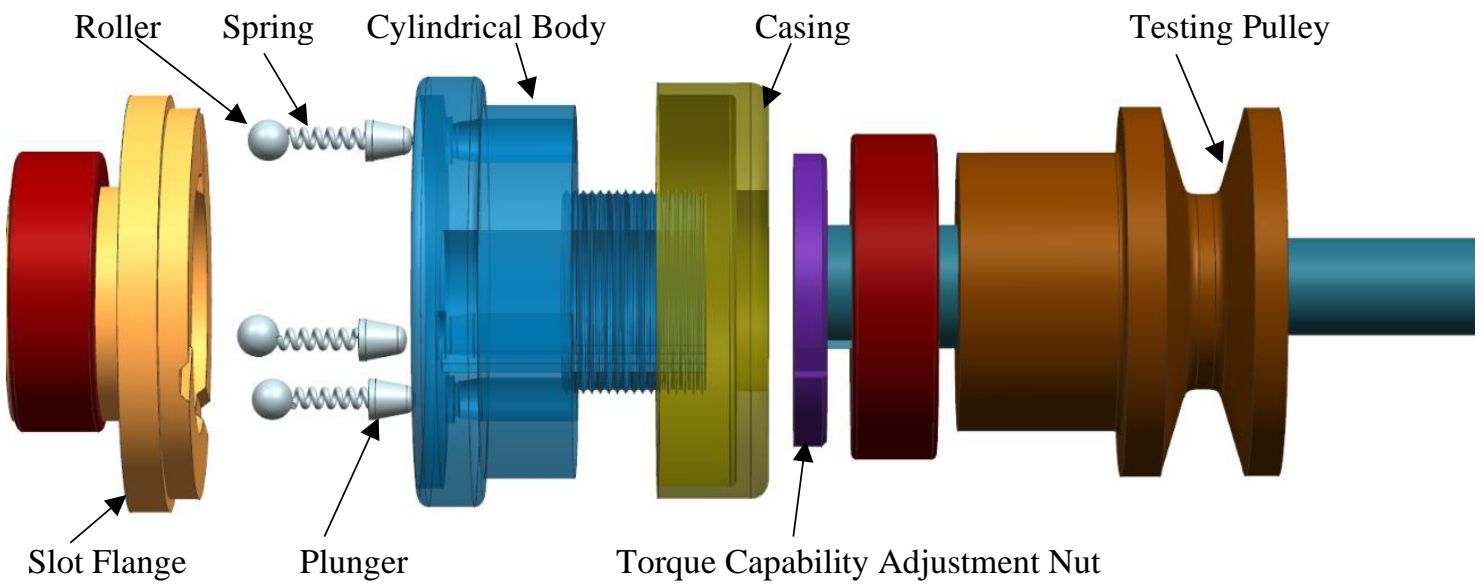

Figure 2: Disassemble View of Overload Protection Roller Clutch 


\section{Scope}

Many of the people can't able to operate a lever-operated clutch system. For its operation, it needs a skilled person while changing gears or stopping, braking of the vehicle. This complication of driving forces to develop a fully automatic transmission system. It observed that automatic transmission is increasing all over the world. Hence the automatically operated clutch market rapidly increases in upcoming years. In some foreign developed countries, automotive clutch systems are well advanced. Because of the very high cost of the present automatic clutch system, a large number of medium and low-class vehicles still use the lever-operated clutch, and they are focusing on an economical automatic transmission for driving simplicity. Some countries like japan South Korea India china are in the position of the high demand for automotive clutches, these countries likely to shift towards the fully automatic transmission.

In some robotics and other machine operation frequency of overloading is high in such a situation, the automatic engage-disengage overload protection clutch is necessary. In some cases, the torque carrying capacity must be variable. These condition forces to develop fully automatic overload protection clutches. After the implementation of the overload protection clutch, the machine secured from overload damage. It saves the breakdown time of machinery and increases the productivity of the organization. By implementing such a small device avoids any future obstructions and reduces the cost of the product. It also provides safety to the operator. Hence these clutches are highly demanded in almost all the industries.

\section{Application, Advantages and Disadvantages}

\subsection{Overload Protection Roller Clutch}

\subsubsection{Application}

Roller clutches used to saves the low torque transmission machine from overload. Due to its simple construction, low cost, easy installation, and low running cost. These clutches used in many types of machinery like the Packing machines Conveyor system, painting industries, food industries rolling mills, robotics, etc.

\subsubsection{Advantages}

1. It provides safety to the operator by disengaging power train

2. Due to the reduction in malfunctioning in machines, the productivity of plants increases causes to reduces the cost of products per piece.

3. The automatic engage-disengagement of the roller clutch avoids manual re-engagement of clutch causes to save operator time.

4. This clutch is reliable, precise and accurate.

5. Zero maintenance, no attention needed.

\subsubsection{Disadvantages}

1. This ball clutch is spring-loaded. Henceforth, they can't use for high torque transmissions machines. If we increase the torque capability of the clutch size of the roller clutch is also increases, which may not fit in compact space.

\subsection{Automatic Electromechanical Single Plate Clutch}




\subsubsection{Application}

Automatic single plate clutches widely used in small vehicles. Due to its ability to disconnect power trains, they also used in mining, manufacturing industries, in mixers in chemical industries to maintain the machine at the desired level and saves motor from overload and burning.

\subsubsection{Advantages}

1. Its engagement is smooth, and no shock recognize.

2. A small slip occurs only during the initial stage of power transmission.

3. Because of slippage during excessive torque than design torque, it gives safety to the machine.

4. Automatic engage and disengage as per need.

5. It eliminates the need of starting devices.

6. Friction clutches can engage-disengage while the machine is running condition.

\subsubsection{Disadvantages}

1. Increasing torque transmitting capacity will also increase the size of the system.

2. A slip occurs during the overload condition.

3. Clutch plate need to change after wear out of friction material.

4. At the initial stage of engaging, slip occurs.

5. Due to frictional force during the slip wear and tear occurred.

6. As soon as wear takes place slippage of cutch also get increased.

\section{Conclusion}

1. It observed that no such two automatic device combinations available in the market make this research unique.

2. Due to the full automation of a single plate clutch and roller clutch, this system may use in a vacuum and hazardous environment.

3. Due to the simple construction, a few parts, and the use of simple manufacturing processes, these two unique systems can be manufacture at economical cost.

4. Because of the parallel construction of spring in roller clutch, torque carrying capability can change by making a combination of different material spring-like aluminum, copper, steel, and also the stiffness of springs can find out by reverse calculation.

\section{Acknowledgment}

First of all, I would like to express my sincere thanks to my respected Project Guide Dr. Badadhe for his enormous assistance \& extended co-operation to me. Finally, I would thank all the workers, operators and other people who have helped me directly and indirectly to make project successful.

\section{References}

[1] Guy James Burlington, Martin David Townsend, "Clutch Mechanism", United States Patent 2005

[2] Charles W. Yeiser, Volker Hütten, Akram Ayoub \& Robert Rheinboldt, "Revamping A Gas Compressor Drive Train From 7000 To 8000 Hp With A New Synchronous Motor Drive And A Controlled Slip Clutch Mechanism”, Proceedings Of The Thirty-Fifth Turbomachinery Symposium 2006. 
[3] Nicolae Eftimie, "Dynamic Simulation of The Safety Clutches With Balls", Transilvania University of Brasov Faculty of Technological Engineering Romania 2007.

[4] Duane W. Woltjen, “Torque Limiting Overload Coupling”, United States Patent 1977.

[5] Donald. Miller, Horseheads. N. Y, "Electric Overload Clutch", United States Patent 1971.

[6] Thomas C. Kilwin, “Torque Limiting Clutch", United States patent 1988.

[7] www.ime.rwth-aachen.de/index.php

[8] Andrew Lechner, "Servo Rated Ball-Detent Torque Limiter" Design news (September-2013 Issue).

[9] R. P. G. Heath and A. J. Child, 'Zeroshift Automated Manual Transmission (AMT)', SAE Paper No. 200726-061, (2007), pp.693-696, 2007.

[10] D Simmer, 'The contribution of Transmission to vehicle fuel economy', AUTOTECH, volume 34, pp.135$145,1995$.

[11] Wang 1,* Richard James Housden 1, Yohan Noh 1, Anisha Singh 2, Lukas Lindenroth 3, Hongbin Liu 3, Kaspar Althoefer ${ }^{4}$, Joseph Hajnal 1 Davinder Singh 2 and Kawal Rhode. "Analysis of a Customized Clutch Joint Designed for the Safety Management of an Ultrasound Robot” Article in Applied Sciences May 2019 